\title{
Quantitative Cerebral Perfusion Imaging in Children and Young Adults with Moyamoya Disease: Comparison of Arterial Spin-Labeling-MRI and $\mathrm{H}_{2}\left[{ }^{15}\right.$ O]-PET
}

\author{
R. Goetti, G. Warnock, F.P. Kuhn, R. Guggenberger, R. O'Gorman, A. Buck, N. Khan, and I. Scheer
}

\begin{abstract}
BACKGROUND AND PURPOSE: Cerebral perfusion assessment is important in the preoperative evaluation and postoperative follow-up of patients with Moyamoya disease. The objective of this study was to evaluate the correlation of quantitative CBF measurements performed with arterial spin-labeling-MR imaging and $\mathrm{H}_{2}\left[{ }^{[5} \mathrm{O}\right]-\mathrm{PET}$ in children and young adults with Moyamoya disease.
\end{abstract}

MATERIALS AND METHODS: Thirteen children and young adults (8 female patients; age, $9.7 \pm 7.1$ years; range, 1-23 years) with Moyamoya disease underwent cerebral perfusion imaging with $\mathrm{H}_{2}\left[{ }^{15} \mathrm{O}\right]$-PET (Discovery STE PET/CT, 3D Fourier rebinning filtered back-projection, $128 \times 128 \times 47$ matrix, $2.34 \times 2.34 \times 3.27 \mathrm{~mm}^{3}$ voxel spacing) and arterial spin-labeling (3T scanner, 3D pulsed continuous arterial spin-labeling sequence, 32 axial sections, $\mathrm{TR}=5.5$ seconds, $\mathrm{TE}=25 \mathrm{~ms}, \mathrm{FOV}=24 \mathrm{~cm}, 128 \times 128$ matrix, $1.875 \times 1.875 \times 5 \mathrm{~mm}^{3}$ voxel spacing) within less than 2 weeks of each other. Perfusion of left and right anterior cerebral artery, MCA, and posterior cerebral artery territories was qualitatively assessed for arterial spin-labeling-MR imaging and $\mathrm{H}_{2}\left[{ }^{15} \mathrm{O}\right]-\mathrm{PET}$ by 2 independent readers by use of a 3-point-Likert scale. Quantitative correlation of relative CBF with cerebellar normalization between arterial spin-labeling-MR imaging and $\mathrm{H}_{2}\left[{ }^{15} \mathrm{O}\right]-\mathrm{PET}$ was evaluated in a volume-based approach for each vascular territory after 3D image coregistration.

RESULTS: Interreader agreement was good ( $\kappa=0.67-0.69)$, and strong and significant correlations were found between arterial spinlabeling-MR imaging and $\mathrm{H}_{2}\left[{ }^{15} \mathrm{O}\right]-\mathrm{PET}$ for both qualitative perfusion scoring $(\rho=0.77 ; P<.001)$ and quantitative perfusion assessment of relative CBF with cerebellar normalization $(r=0.67, P<.001)$.

CONCLUSIONS: In children and young adults with Moyamoya disease, quantitative evaluation of CBF is possible with the use of arterial spin-labeling-MR imaging without ionizing radiation or contrast injection with a good correlation to $\mathrm{H}_{2}\left[{ }^{15} \mathrm{O}\right]-\mathrm{PET}$ after cerebellar normalization.

ABBREVIATIONS: $\mathrm{ASL}=$ arterial spin-labeling; $\mathrm{rCBF}=$ relative $\mathrm{CBF}$

$\mathrm{C}_{8}^{\mathrm{h}}$ hildren and young adults with Moyamoya disease have progressive bilateral stenosis of the supraclinoid ICA, anterior cerebral artery, MCA, and to a much lesser extent, the posterior cerebral artery, with formation of netlike collateral vessel networks, termed "Moyamoya" vessels. " "Moyamoya" is the Japanese term for "puff of smoke" and describes the characteristic angiographic appearance of the collateral vessel networks typically formed at the skull base and around the basal ganglia in

Received August 27, 2013; accepted after revision September 25.

From the Departments of Diagnostic Imaging (R. Goetti, R. Guggenberger, I.S.), Diagnostic and Interventional Radiology (R. Goetti, F.P.K., R. Guggenberger), and Nuclear Medicine (G.W., F.P.K., A.B.), Center for MR Research (R.O.); and the Moyamoya Center, Division of Neurosurgery, Department of Surgery (N.K.), University Children's Hospital Zurich, Zurich, Switzerland.

Please address correspondence to Robert Goetti, MD, Department of Diagnostic and Interventional Radiology, University Hospital Zurich, Raemistr 100, CH-8091

Zurich, Switzerland; e-mail: robertpaul.goetti@usz.ch

http://dx.doi.org/10.3174/ajnr.A3799
Moyamoya disease. The condition is rare; however, it is implicated in approximately $6 \%$ of childhood strokes. ${ }^{2}$ The main goal of treatment of children with Moyamoya is the prevention of future strokes by means of surgical revascularization.

To determine the optimal targets for revascularization procedures as well as for postoperative follow-up, CBF studies with the use of Xe-CT, SPECT, or PET have been shown to be of high clinical value. ${ }^{3-7}$ However, these methods are associated with ionizing radiation, which should be minimized or avoided whenever possible and especially in young patients. Recently, interest in MR imaging techniques for CBF assessment has grown, and initial evaluation of these techniques has been undertaken in patients with Moyamoya disease. MR perfusion imaging by use of dynamic susceptibility contrast imaging, on the basis of the decrease of $\mathrm{T} 2 / \mathrm{T}^{*}$ times of tissue during the first pass of a gadoliniumcontaining contrast agent through the cerebral capillary bed, has been shown to correlate well with the reference standard of 
$\mathrm{H}_{2}\left[{ }^{15} \mathrm{O}\right]-\mathrm{PET}^{8}$ in the assessment of cerebrovascular occlusive disease. Arterial spin-labeling (ASL) is an alternative MR imaging technique for CBF imaging that is based on the T1 magnetization state of electromagnetically tagged (ie, spin-labeled), freely diffusible arterial water. Blood flow measurements with ASL have been shown to correlate well with those from DSC imaging in children with Moyamoya disease. ${ }^{9}$

As a method requiring neither ionizing radiation nor exogenous contrast material injection, ASL could represent an ideal technique for the assessment of cerebral perfusion in children. The purpose of the present study was thus to assess the comparability of ASL and the reference standard of $\mathrm{H}_{2}\left[{ }^{15} \mathrm{O}\right]$-PET imaging in children and young adults with Moyamoya disease.

\section{MATERIALS AND METHODS \\ Patients}

Children and young adults with angiographically proven Moyamoya disease who underwent both MR imaging with ASL imaging as well as $\mathrm{H}_{2}\left[{ }^{15} \mathrm{O}\right]$-PET for preoperative assessment or postoperative follow-up after surgical revascularization as a routine work-up at our institutions between January 2011 and December 2012 were included in this retrospective study. The informed consent requirement was waived by the institutional review board because of the retrospective nature of the study.

Exclusion criteria were patient age $>25$ years $(n=0)$, $\mathrm{H}_{2}\left[{ }^{15} \mathrm{O}\right]$-PET not performed within 2 weeks of ASL $(n=11)$, interventions or cerebrovascular incidents (ie, transient ischemic attacks or strokes) between the 2 examinations $(n=0)$, postoperative status $<6$ months $(n=0)$, contraindications to sedation in children $<6$ years old as evaluated by a board-certified anesthesiologist $(n=0)$, contraindications to contrast-enhanced MR imaging, such as noncompatible metallic implants or known previous allergic reactions to gadolinium-based contrast material $(n=0)$, and nondiagnostic image quality of either ASL or $\mathrm{H}_{2}\left[{ }^{15} \mathrm{O}\right]$-PET studies $(n=1$ : nondiagnostic image quality of ASL caused by motion artifacts).

Thirteen children and young adults ( 8 female patients and 5 male patients; age, $9.7 \pm 7.1$ years; range, $1-23$ years) met all criteria and were thus included in the study. Suzuki stages of the angiographically confirmed Moyamoya disease in these patients were II $(n=4)$, III $(n=4), \operatorname{IV}(n=3)$, and V $(n=2)$. Anterior watershed infarctions were present in 4 patients unilaterally and in 1 patient bilaterally. The mean time interval between the 2 examinations was $2 \pm 3$ days (range, $1-13$ days). $\mathrm{H}_{2}\left[{ }^{15} \mathrm{O}\right]$-PET was performed first in 3 patients, whereas ASL was performed first in 10 patients. Because of the retrospective nature of the study, the order of acquisition was not randomized.

\section{MR Imaging Protocol and Image Reconstruction}

MR imaging was performed with the use of a 3T scanner (HD.xt TwinSpeed; GE Healthcare, Milwaukee, Wisconsin) equipped with an 8-channel receive-only head coil for signal reception in all patients. Patients under the age of 6 years $(n=6)$, were sedated with propofol (Propofol-Lipuro; Braun Medical, Melsungen, Germany) and monitored by a board-certified anesthesiologist during the examination. A standardized MR imaging protocol for patients with Moyamoya disease consisting of multiplanar T2WI,
T1WI, FLAIR, DWI, 3D-TOF, ASL, and contrast-enhanced DSC as well as multiplanar postcontrast T1WI was performed in all patients without complications.

ASL images were acquired as 32 axial sections by use of a background-suppressed, pulsed continuous arterial spin-labeling sequence, with a 3D stack of spiral fast spin-echo readout. ${ }^{10}$ The labeling duration was 1.5 seconds, and a postlabeling delay of 1.5 seconds was used to reduce errors from transit time effects. ${ }^{11}$ Further imaging parameters of the ASL sequence were TR, $5500 \mathrm{~ms}$; TE, $25 \mathrm{~ms}$; FOV, $24 \mathrm{~cm}$; matrix, $128 \times 128$; section thickness, 5 $\mathrm{mm}$; and number of excitations, 3 . The reconstructed voxel spacing was $1.875 \times 1.875 \times 5 \mathrm{~mm}^{3}$. The total scan time was $5 \mathrm{~min}$ utes, 34 seconds.

Quantitative CBF maps were calculated from the ASL data by use of the standard on-line perfusion image reconstruction provided by the scanner manufacturer. This reconstruction calculates the perfusion images according to the model described by Wang et $\mathrm{al},{ }^{12}$ with an additional factor included to correct for incomplete recovery of the magnetization in the reference image caused by a saturation pulse applied 2 seconds before imaging. ${ }^{13}$ No further preprocessing was performed. CBF was calculated according to the following equation. ${ }^{11,13}$

$$
f=\frac{\lambda \quad\left(S_{\mathrm{ctrl}}-S_{\mathrm{lbl}}\right)\left(1-e^{-\frac{t_{\mathrm{sat}}}{T_{\mathrm{lg}}}}\right)}{2 \alpha T_{1 \mathrm{~b}}\left(1-e^{-\frac{\tau}{T_{\mathrm{bb}}}}\right)} e^{\frac{w}{T_{\mathrm{ref}}}}
$$

In this equation, $f$ is blood flow (in $\mathrm{mL} / \mathrm{min}$ per $100 \mathrm{mg}$ ), $S_{\mathrm{ctrl}}-S_{\mathrm{lbl}}$ is the difference image (control-label), and $S_{\text {ref }}$ is a proton-attenuation-weighted reference image; $\lambda$ is the blood-brain partition coefficient (0.9), $\alpha$ is the inversion efficiency, $T_{1 \mathrm{~b}}$ is the T1 of blood (1600 $\mathrm{ms}), T_{1 \mathrm{~g}}$ is the $\mathrm{T} 1$ of gray matter $(1200 \mathrm{~ms}), w$ is the postlabeling delay (1.5 seconds), and $\tau$ is the labeling duration (1.5 seconds). ${ }^{9,12,13}$ To investigate the effects of variations in blood $\mathrm{T} 1$ across the patient group, the blood $\mathrm{T} 1$ was calculated for $n=11$ patients from venous hematocrit levels according to the equation $\mathrm{T} 1=1 /(0.83 \mathrm{Hct}+0.28)$. ASL perfusion values were calculated by use of an assumed blood T1 of $1600 \mathrm{~ms}$ and by use of the individual blood T1 for the patients in whom hematocrit data were available.

\section{PET Protocol and Image Reconstruction}

The PET data were acquired in 3D mode on a full-ring PET/CT scanner (Discovery STE; GE Healthcare) and corrected for attenuation, scatter, random photons, and dead time by use of the corresponding low-dose CT $(120 \mathrm{kV} / 80 \mathrm{~mA})$. The scanner's axial FOV covered $15.3 \mathrm{~cm}$, and transaxial images were reconstructed by use of a 3D Fourier rebinning filtered back-projection algorithm resulting in a $128 \times 128 \times 47$ matrix with $2.34 \times 2.34 \times$ $3.27 \mathrm{~mm}^{3}$ voxel spacing; $400-800 \mathrm{MBq}_{2}\left[{ }^{15} \mathrm{O}\right]$ was administered intravenously by use of an automatic injection device that delivered the predefined tracer dose over a period of 20 seconds. Patients under the age of 6 years ( $n=6$, the same as in ASL) were sedated with the use of Propofol-Lipuro and monitored by a boardcertified anesthesiologist during the examination. The emission data were acquired as a series of eighteen, 10-second frames.

Parametric images of CBF were generated by means of a reported method ${ }^{14}$ in which a standardized arterial input function 


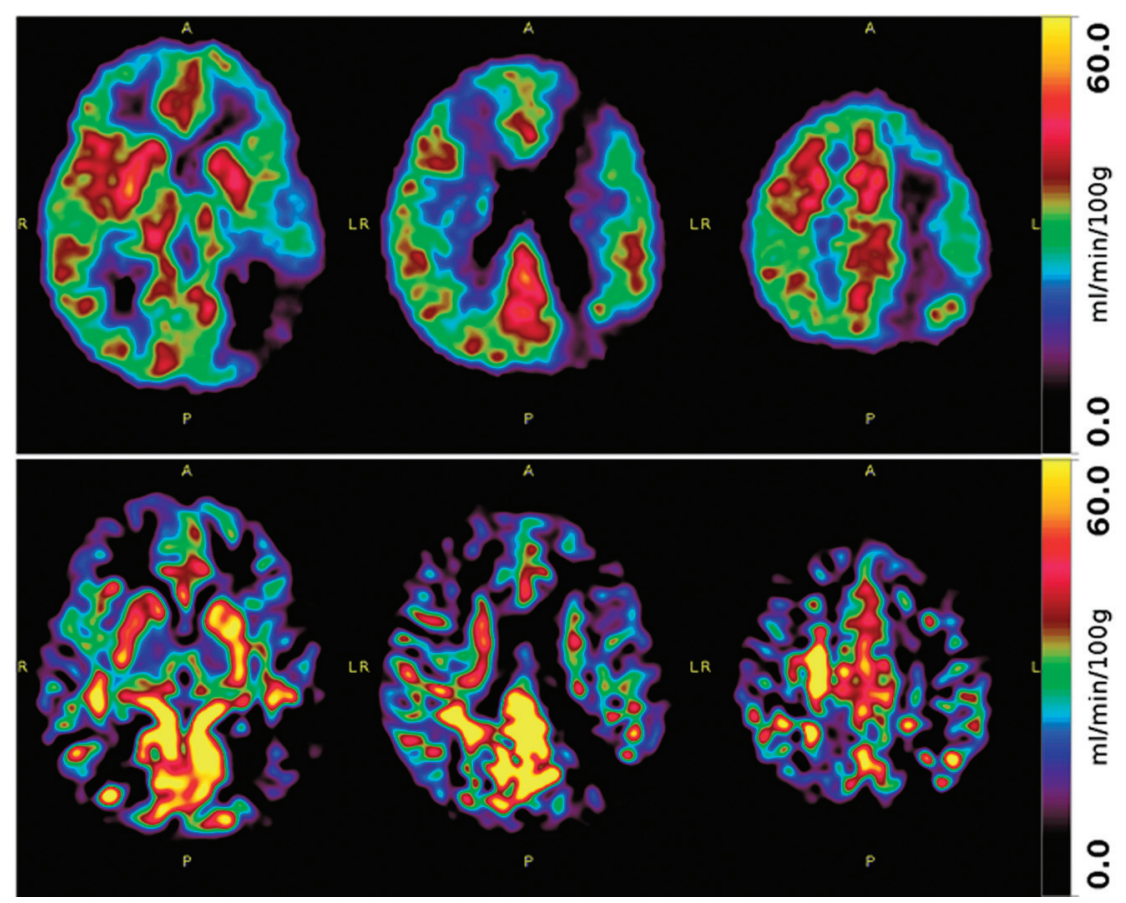

FIG 1. Example of $\mathrm{CBF}$ maps from $\mathrm{H}_{2}\left[{ }^{15} \mathrm{O}\right]-\mathrm{PET}$ (upper row) and ASL (lower row) in the same 2-year-old girl with Moyamoya disease show reduced perfusion in the bilateral anterior and middle cerebral artery territories, more pronounced on the left (score 3) than on the right (score 2).

and image scaling on the basis of the washout rate $(\mathrm{k} 2)$ of $\mathrm{H}_{2}\left[{ }^{15} \mathrm{O}\right]$ are used to derive CBF. This technique, which is based on the Alpert method, exploits the fact that $\mathrm{k} 2$ is related to the shape and not the scale of the arterial input function and proportional to relative $\mathrm{CBF}(\mathrm{rCBF}):(\mathrm{k} 2=\mathrm{K} 1 / \mathrm{p}$, where $\mathrm{p}$ is the partition coefficient). ${ }^{15}$

\section{MR Imaging and PET Image Analysis}

Qualitative Analysis. ASL and $\mathrm{H}_{2}\left[{ }^{15} \mathrm{O}\right]$-PET CBF maps were assessed separately by 2 blinded and independent radiologists with 5 years and 7 years of experience. CBF was visually categorized on a 3-point Likert scale (1: normal, 2: reduced, 3: severely reduced) in the color-coded CBF maps (Fig 1) of each technique for anterior cerebral artery, MCA, and posterior cerebral artery territories on each side by comparison to cerebellar perfusion, which was assumed to be normal.

Quantitative Image Analysis. Semi-automated image analysis was performed in PMOD (version 3.4; PMOD Technologies, Zurich, Switzerland). With the use of a PET perfusion template image (SPM5, average $\mathrm{H}_{2}\left[{ }^{15} \mathrm{O}\right]$ image from 12 subjects), ASL and PET perfusion maps were normalized to the stereotaxic spatial array of the Montreal Neurological Institute brain template by applying a mutual information-based registration including 12parameter affine and elastic transformations and transitional image smoothing with a 6-mm full width at half maximum Gaussian kernel. Successful normalization was verified visually with the aid of image-outlining contours. After normalization, a volume-ofinterest template defining the vascular territories (left/right, anterior cerebral artery, MCA, and posterior cerebral artery) and cerebellum was used to extract average perfusion by territory.
This volume-of-interest template was generated by attributing the predefined brain regions outlined by the Hammer $\mathrm{N} 30 \mathrm{R} 83$ atlas $^{16}$ to the respective vascular territories and was identical in size and shape for both $\mathrm{H}_{2}\left[{ }^{15} \mathrm{O}\right]$-PET and ASL data (Fig 2). Relative CBF values (rCBF), that is, cerebellar normalized values, were calculated by dividing the CBF value of the respective supratentorial territory by cerebellar CBF.

\section{Statistical Analysis}

Cohen $\kappa$ statistics were used for the assessment of interreader agreement in the evaluation of qualitative cerebral perfusion. Kappa values were interpreted as follows: $\kappa \geq 0.81$, excellent; $\kappa=0.61-$ 0.80 , good; $\kappa=0.41-0.60$, moderate; $\kappa=$ $0.21-0.40$, fair; and $\kappa \leq 0.20$, poor agreement.

The correlation between $\mathrm{H}_{2}\left[{ }^{15} \mathrm{O}\right]$ PET and ASL was assessed by use of Spearman rank correlation coefficient for qualitative perfusion scores and by Pearson correlation coefficient for quantitative $\mathrm{CBF}$ values and quantitative relative $\mathrm{CBF}$ values after cerebellar normalization. Bland-Altman analysis was used to compare mean differences in $\mathrm{CBF}$ and $\mathrm{rCBF}$ between $\mathrm{H}_{2}\left[{ }^{15} \mathrm{O}\right]$-PET and ASL.

Paired $t$ tests were used to compare CBF and rCBF values between $\mathrm{H}_{2}\left[{ }^{15} \mathrm{O}\right]$-PET and ASL, and unpaired $t$ tests were used to compare $\mathrm{CBF}$ and $\mathrm{rCBF}$ between territories with normal (qualitative perfusion score of 1 ) and reduced perfusion (score of 2 or 3 ) for both $\mathrm{H}_{2}\left[{ }^{15} \mathrm{O}\right]$-PET and ASL. Differences in CBF and rCBF between territories grouped by perfusion scores were assessed by means of 1-way ANOVA with Tukey honestly significant differences post hoc analysis. All statistical tests were performed with the use of the statistical package $\mathrm{R}^{17}$ (http:// www.r-project.org/) and the R frontend RKWard Version 0.6.1 (http://rkward.sourceforge.net/). Statistical significance was inferred at $P<.05$.

\section{RESULTS}

\section{Qualitative Image Analysis}

Interreader agreement for qualitative perfusion assessment was good for both $\mathrm{H}_{2}\left[{ }^{15} \mathrm{O}\right]$-PET $(\kappa=0.67)$ and ASL $(\kappa=0.69)$. A strong and significant correlation between perfusion scores in ASL and $\mathrm{H}_{2}\left[{ }^{15} \mathrm{O}\right]$-PET was found $(\rho=0.77 ; P<.001)$. No posterior circulation involvement that could have influenced cerebellar normalization was found in our patient cohort.

Reduced perfusion in the visual qualitative assessment was found in 33 of 78 (42\%) territories in 11 of 13 (85\%) patients with $\mathrm{H}_{2}\left[{ }^{15} \mathrm{O}\right]$-PET and in 32 of $78(41 \%)$ territories in the same 11 of $13(85 \%)$ patients with ASL. An overview of the qualitative perfusion assessment per vascular territory in both $\mathrm{H}_{2}\left[{ }^{15} \mathrm{O}\right]$-PET and ASL is provided in the Table. 


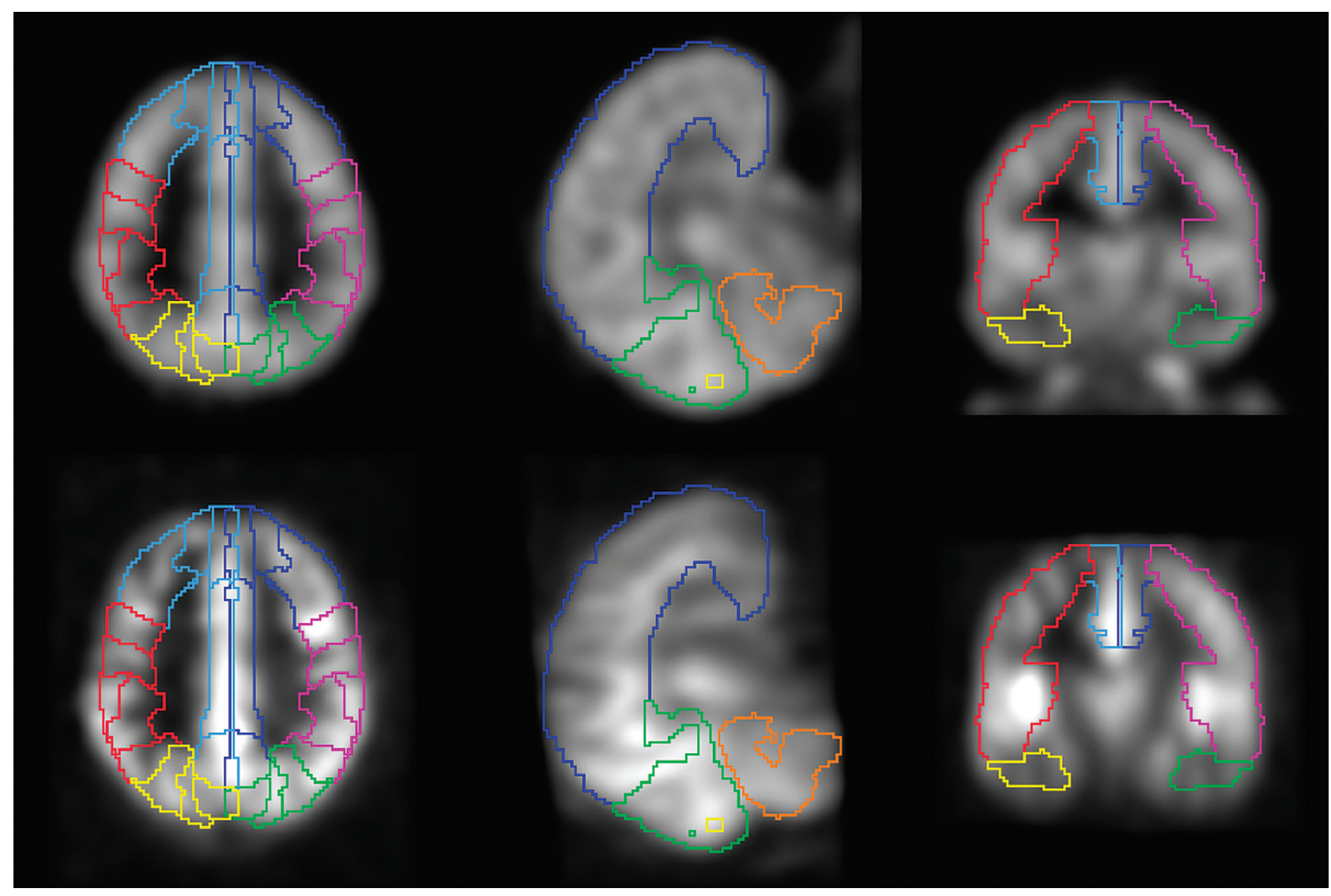

FIG 2. Volume-of-interest definition for quantitative $C B F$ assessment per territory (upper row: $\mathrm{H}_{2}\left[{ }^{15} \mathrm{O}\right]-\mathrm{PET}$; lower row: arterial spin-labeling).

Overview of qualitative perfusion scores and quantitative perfusion in $\mathrm{H}_{2}\left[{ }^{15}\right.$ O $]$-PET and ASL

\begin{tabular}{|c|c|c|c|c|}
\hline \multirow{2}{*}{$\begin{array}{l}\text { Qualitative } \\
\text { Perfusion }\end{array}$} & \multirow{2}{*}{$\begin{array}{c}\text { Quantitative } \\
\text { Perfusion }\end{array}$} & \multicolumn{3}{|c|}{ Territory } \\
\hline & & ACA & MCA & PCA \\
\hline \multicolumn{5}{|l|}{ PET } \\
\hline Normal & $\begin{array}{l}C B F: 31.51 \pm 6.87^{a} \\
\text { rCBF: } 1.011 \pm 0.112\end{array}$ & $9 / 26(35 \%)$ & $13 / 26(50 \%)$ & $23 / 26(88 \%)$ \\
\hline Reduced & $\begin{array}{l}\text { CBF: } 28.20 \pm 6.97^{\mathrm{a}} \\
\text { rCBF: } 0.943 \pm 0.163\end{array}$ & $12 / 26(46 \%)$ & $9 / 26(35 \%)$ & $3 / 26(12 \%)$ \\
\hline Severely Reduced & $\begin{array}{l}\text { CBF: } 23.00 \pm 3.70^{a} \\
\text { rCBF: } 0.677 \pm 0.161\end{array}$ & $5 / 26(19 \%)$ & $4 / 26(15 \%)$ & \\
\hline \multicolumn{5}{|l|}{ ASL } \\
\hline Normal & $\begin{array}{l}\text { CBF: } 31.88 \pm 8.24^{a} \\
\text { rCBF: } 1.059 \pm 0.159\end{array}$ & $6 / 26(23 \%)$ & $15 / 26(57 \%)$ & $25 / 26(96 \%)$ \\
\hline Reduced & $\begin{array}{l}\text { CBF: } 24.76 \pm 7.58^{a} \\
\text { rCBF: } 0.853 \pm 0.249\end{array}$ & $15 / 26(58 \%)$ & $8 / 26(31 \%)$ & $1 / 26(4 \%)$ \\
\hline Severely Reduced & $\begin{array}{l}\text { CBF: } 16.06 \pm 5.18^{\mathrm{a}} \\
\text { rCBF: } 0.507 \pm 0.098\end{array}$ & $5 / 26(19 \%)$ & $3 / 26(12 \%)$ & None \\
\hline
\end{tabular}

Note:-ACA indicates anterior cerebral artery; PCA, posterior cerebral artery; rCBF, relative CBF (values are given as ratios to cerebellar $\mathrm{CBF}$ ).

${ }^{a} \mathrm{CBF}$ values are given as $\mathrm{mL} / \mathrm{min}$ per $100 \mathrm{~g}$.

\section{Quantitative Image Analysis}

Quantitative image analysis revealed a strong and significant correlation between $\mathrm{H}_{2}\left[{ }^{15} \mathrm{O}\right]$-PET and ASL $(r=0.67, P<.001)$ after cerebellar normalization ( $\mathrm{rCBF}$ ), with a regression slope of 1.0061 and intercept of -0.0057 (Fig 3). For absolute CBF values, the correlation was much weaker $(r=0.26, P=.015)$ using a literature value (1600 ms) for the blood T1 in the ASL perfusion quantification. The correlation was not improved with the use of an individual blood T1 on the basis of the hematocrit $(r=0.14, P=.025)$.
Mean CBF values (across all perfusion territories) were $30.58 \pm 7.56 \mathrm{~mL} / \mathrm{min}$ per $100 \mathrm{~g}$ in $\mathrm{H}_{2}\left[{ }^{15} \mathrm{O}\right]$-PET and $28.38 \pm 9.15$ $\mathrm{mL} / \mathrm{min}$ per $100 \mathrm{~g}$ in ASL. Bland-Altman analysis showed a mean difference of $2.19 \pm 10.23 \mathrm{~mL} / \mathrm{min}$ per $100 \mathrm{~g}$ between $\mathrm{H}_{2}\left[{ }^{15} \mathrm{O}\right]$-PET and ASL $(P=.062)$. After cerebellar normalization, mean rCBF values (all territories) were $0.958 \pm 0.165$ for $\mathrm{H}_{2}\left[{ }^{15} \mathrm{O}\right]$-PET and $0.951 \pm 0.247$ for ASL, with a mean difference of $0.007 \pm 0.186$ $(P=.737)$ (Fig 4).

Territories with normal qualitative perfusion (score 1) showed similar values in $\mathrm{H}_{2}\left[{ }^{15} \mathrm{O}\right]$-PET and ASL $(P=.497)$. Similar values were also found for territories with reduced perfusion (score 2 ) in $\mathrm{H}_{2}\left[{ }^{15} \mathrm{O}\right]$-PET and ASL $(P=.162)$. For territories with severely reduced perfusion (score 3), values tended to be higher in $\mathrm{H}_{2}\left[{ }^{15} \mathrm{O}\right]$-PET (CBF: $23.00 \pm 3.70 \mathrm{~mL} / \mathrm{min}$ per $100 \mathrm{~g}$; rCBF: $0.677 \pm 0.161$ ) than in ASL (CBF: $16.06 \pm 5.18 \mathrm{~mL} / \mathrm{min}$ per $100 \mathrm{~g}$; rCBF: $0.507 \pm 0.098$ ), though not at a statistically significant level $(P=.085)$. An overview of all quantitative perfusion values is provided in the Table. Quantitative perfusion values were significantly lower in territories with reduced qualitative perfusion scores (ie, scores 2 and 3 ) compared with those with normal qualitative perfusion for both $\mathrm{H}_{2}\left[{ }^{15} \mathrm{O}\right]$-PET (CBF: $P=.025$, rCBF: 
$P=.016)$ and ASL (CBF: $P=.010, \mathrm{rCBF}: P=.004)$. Significant differences between territories with reduced perfusion (score 2 ) and severely reduced perfusion (score 3 ) were found for ASL $(P=$ $.03)$ but not for $\mathrm{H}_{2}\left[{ }^{15} \mathrm{O}\right]-\mathrm{PET}(P=.17)$.

\section{DISCUSSION}

In the present study, we compared MR imaging-based and PETbased methods for the evaluation of cerebral perfusion in children and young adults with Moyamoya disease, through the use of qualitative and quantitative approaches. Our results demonstrate that CBF assessment by use of ASL-MR imaging with cerebellar

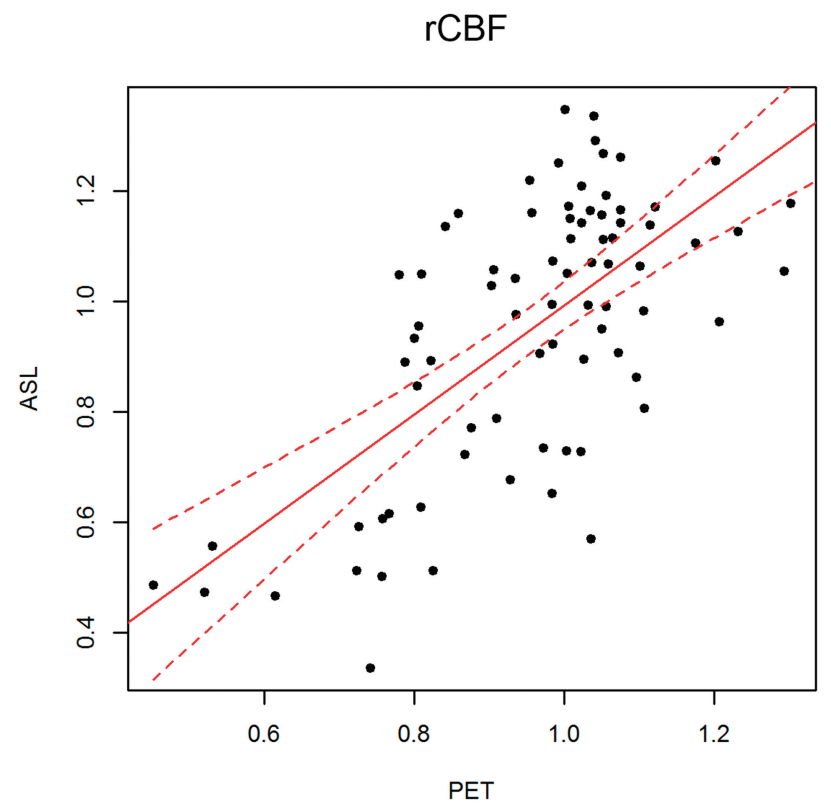

FIG 3. Correlation of relative $\mathrm{CBF}$ after cerebellar normalization (rCBF) between $\mathrm{H}_{2}\left[{ }^{15} \mathrm{O}\right]-P E T$ and arterial spin-labeling $(r=0.67, P<$ .001). Linear regression fit line is shown in red (dashed lines show $95 \%$ confidence limits).

\section{CBF $($ difference $=$ ASL - PET $)$}

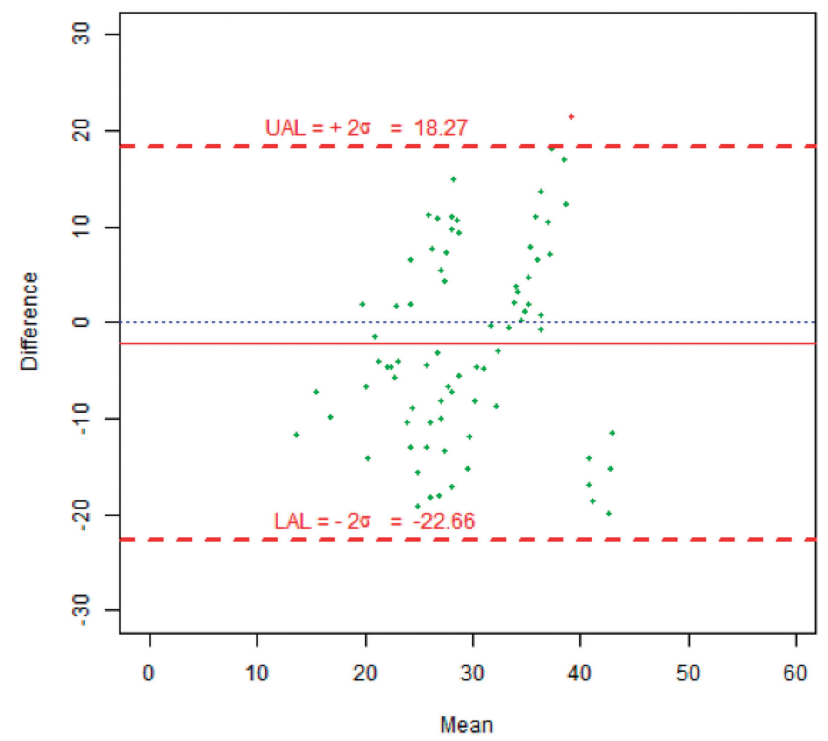

normalization correlates well with the reference standard $\mathrm{H}_{2}\left[{ }^{15} \mathrm{O}\right]$-PET method. This suggests that ASL-MR imaging could be a valuable alternative to $\mathrm{H}_{2}\left[{ }^{15} \mathrm{O}\right]$-PET, while avoiding the use of ionizing radiation and injection of an exogenous contrast agent. However, absolute values of CBF may differ between the modalities.

The most widely used MR imaging technique is gadoliniumenhanced DSC, which is based on the decrease of $\mathrm{T} 2 / \mathrm{T} 2{ }^{*}$ signal intensity of cerebral tissue during the first pass of gadolinium through the capillary bed. It has been shown to reveal alterations in perfusion even in apparently (structurally) normal cerebral parenchyma in patients with Moyamoya disease and may be used in the targeting of revascularization approaches. ${ }^{18}$ In a recent study, quantitative $\mathrm{CBF}$ values obtained with DSC were shown to strongly correlate with $\mathrm{CBF}$ values from $\mathrm{H}_{2}\left[{ }^{15} \mathrm{O}\right]$-PET in patients with Moyamoya disease or ICA occlusions, though with a slight underestimation of CBF values in DSC. ${ }^{8}$

Arterial spin-labeling is a well-established method for the quantitative assessment of cerebral perfusion. It does not require the injection of an exogenous contrast agent but instead uses the water in arterial blood as an endogenous tracer through electromagnetic tagging with radiofrequency pulses. For the calculation of blood flow, the amount of labeled blood arriving in the cerebral tissue after a predefined transit time is measured by subtraction from a previously acquired control image. ${ }^{19} \mathrm{~A}$ high correlation between ASL and DSC has been reported, with correlation coefficients between ASL and DSC of 0.62-0.79 in patients with acute ischemic stroke. ${ }^{20}$ In a recent study with the use of DSC as the standard of reference in children with Moyamoya disease, it could be demonstrated that ASL-based measurements of CBF showed a similarly strong and significant correlation with DSC-based perfusion ( $r=0.79, P<.001)$, enabling the detection of impaired perfusion per cerebral vascular territory with a high diagnostic accuracy. ${ }^{9}$ In a direct comparison of ASL and $\mathrm{H}_{2}\left[{ }^{15} \mathrm{O}\right]$-PET, one study found similar cortical perfusion values with no significant

rCBF (difference $=$ ASL - PET)

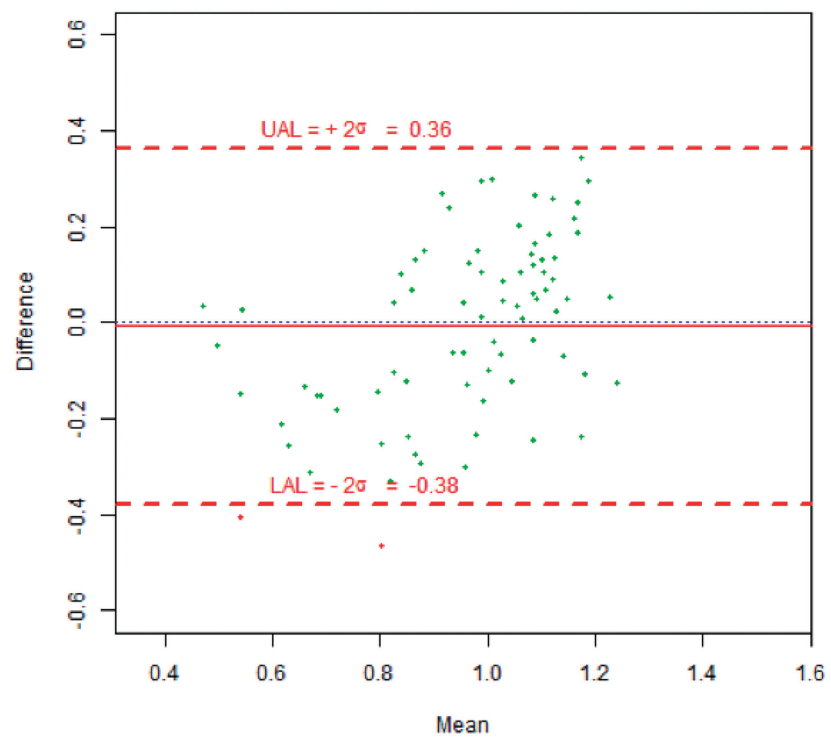

FIG 4. Bland-Altman plots of $\mathrm{CBF}(\mathrm{mL} / 100 \mathrm{~g}$ per minute) and relative $\mathrm{CBF}$ after cerebellar normalization (rCBF). 
differences between the 2 modalities in 12 healthy subjects. ${ }^{21}$ However, to the best of our knowledge, there has been no study comparing cerebral perfusion imaging with ASL and the current reference standard of $\mathrm{H}_{2}\left[{ }^{15} \mathrm{O}\right]$-PET in children and young adults with Moyamoya disease.

Assessment of CBF with ASL imaging has been shown to correlate with ( ${ }^{123}$ I-IMP)-SPECT in patients with Moyamoya disease, though the absolute values of CBF were found to be slightly lower in ASL. ${ }^{22}$ Our results show a similar trend toward underestimation of CBF in ASL compared with $\mathrm{H}_{2}\left[{ }^{15} \mathrm{O}\right]$-PET, particularly in territories with severely reduced perfusion in the qualitative visual assessment. This could be partly due to long transit times caused by the presence of collateral vessel networks feeding these territories, leading to a loss of perfusion signal in which the labeled blood does not arrive between the time of labeling and image acquisition, resulting in artificially low CBF values. ${ }^{23} \mathrm{How}-$ ever, because transit time errors would not be expected to affect the cerebellar perfusion in Moyamoya disease, the observation that cerebellar normalization improves the correlation between ASL and PET suggests that lengthened transit times are not the major source of the discrepancy in absolute perfusion values between the 2 modalities. Rather, our observation that cerebellar normalization improves the agreement between ASL and PET suggests that errors in global scaling factors (which would affect the cerebellum and cortical/subcortical regions equally) are likely to underlie the differences in absolute ASL and PET perfusion values. Because the reliability of ASL perfusion values is most affected by errors in the labeling efficiency, the equilibrium magnetization of blood and tissue, and the blood T1 used for perfusion scaling, ${ }^{24}$ errors in these factors may contribute to the difference in perfusion values measured between ASL and PET observed in the present study. However, accounting for differences in blood T1 did not improve the correlation between ASL and PET perfusion values in our study, suggesting that the discrepancy observed between the perfusion measurements from the 2 modalities without cerebellar normalization are likely to arise from other effects. Future studies should investigate if MR angiographic flow measurements can reduce these errors by correcting the labeling efficiency values used for quantification.

Alternatively, the difference in ASL-derived and $\mathrm{H}_{2}\left[{ }^{15} \mathrm{O}\right]-$ PET-derived CBF values could also be caused by the use of a mean arterial input function-based method for $\mathrm{H}_{2}\left[{ }^{15} \mathrm{O}\right]-\mathrm{PET} .{ }^{14}$ Whereas this method substantially reduces the invasive nature of $\mathrm{H}_{2}\left[{ }^{15} \mathrm{O}\right]$-PET, it does not measure the individual activity in arterial blood. Indeed, CBF measured with the less invasive technique was shown to overestimate true perfusion by approximately $11 \%$. Because this difference is substantially larger than that between ASL and PET in our study, the underestimation that we found could be considered negligible. This is supported by the lack of a significant difference in $\mathrm{CBF}$ or $\mathrm{rCBF}$ in territories with qualitatively normal perfusion.

Generally, comparisons of quantitative values between different modalities with different quantification models must be interpreted with caution. To minimize bias caused by different quantification models, we normalized the CBF values found in the supratentorial vascular territories to cerebellar CBF (rCBF). In patients with Moyamoya disease, which predominantly affects the anterior circulation, cerebellar perfusion may be assumed to be normal and act as an intraindividual reference for the detection of reduced perfusion. In our study, the mean difference in perfusion values between $\mathrm{H}_{2}\left[{ }^{15} \mathrm{O}\right]$-PET and ASL was reduced from approximately $13 \%$ to a mere $0.7 \%$ after cerebellar normalization, leading to a regression slope of 1.0061 (intercept of -0.0057) and allowing for a more meaningful comparison between the 2 modalities.

\section{Limitations}

A limitation of our study is the rather small study cohort. However, this is inherent to the low prevalence of this disease. Second, ASL and $\mathrm{H}_{2}\left[{ }^{15} \mathrm{O}\right]$-PET were not performed in an integrated $\mathrm{PET} / \mathrm{MR}$ imaging scanner in the same examination or on the same day, but up to 13 days apart. However, there were no interventions or cerebrovascular incidents between the 2 examinations in any of the patients. Because we had no patients with both preoperative and postoperative examinations with both ASL and $\mathrm{H}_{2}\left[{ }^{15} \mathrm{O}\right]$-PET, we could not evaluate the correlations of ASL and $\mathrm{H}_{2}\left[{ }^{15} \mathrm{O}\right]$-PET for the assessment of postoperative improvement of perfusion.

\section{CONCLUSIONS}

Our results demonstrate that the assessment of CBF after cerebellar normalization by use of arterial spin-labeling MR imaging correlates well with the $\mathrm{H}_{2}\left[{ }^{15} \mathrm{O}\right]$-PET reference standard method in children and young adults with Moyamoya disease. Therefore, ASL may prove to be a valuable alternative for preoperative assessment and postoperative follow-up in these patients without the use of ionizing radiation or the need for injection of an exogenous contrast agent.

Disclosures: Geoffrey Warnock—OTHER RELATIONSHIPS: Teaching consultant for PMOD Technologies. Alfred Buck—UNRELATED: Royalties: Medrad Inc.

\section{REFERENCES}

1. Suzuki J, Takaku A. Cerebrovascular "Moyamoya” disease: disease showing abnormal net-like vessels in base of brain. Arch Neurol 1969;20:288-99

2. Smith ER, Scott RM. Spontaneous occlusion of the circle of Willis in children: pediatric Moyamoya summary with proposed evidencebased practice guidelines: a review. J Neurosurg Pediatr 2012;9: 353-60

3. Feole JB, Ali A, Fordham EW, et al. Serial SPECT imaging in Moyamoya using I-123 IMP: a method of noninvasive evaluation and follow-up. Clin Nucl Med 1993;18:43-45

4. Ikezaki K, Matsushima T, Kuwabara Y, et al. Cerebral circulation and oxygen metabolism in childhood Moyamoya disease: a perioperative positron emission tomography study. J Neurosurg 1994;81: 843-50

5. Nambu K, Suzuki R, Hirakawa K. Cerebral blood flow: measurement with xenon-enhanced dynamic helical CT. Radiology 1995; 195:53-57

6. Nariai T, Suzuki R, Hirakawa K, et al. Vascular reserve in chronic cerebral ischemia measured by the acetazolamide challenge test: comparison with positron emission tomography. AJNR Am J Neuroradiol 1995;16:563-70

7. Weber B, Westera G, Treyer V, et al. Constant-infusion H(2)150 PET and acetazolamide challenge in the assessment of cerebral perfusion status. J Nucl Med 2004;45:1344-50

8. Vakil P, Lee JJ, Mouannes-Srour JJ, et al. Cerebrovascular occlusive disease: quantitative cerebral blood flow using dynamic suscepti- 
bility contrast MR imaging correlates with quantitative $\mathrm{H} 2[15 \mathrm{O}$ ] PET. Radiology 2013;266:879-86

9. Goetti R, O'Gorman R, Khan N, et al. Arterial spin labelling MRI for assessment of cerebral perfusion in children with Moyamoya disease: comparison with dynamic susceptibility contrast MRI. Neuroradiology 2013;55:639-47

10. Dai W, Garcia D, de Bazelaire C, et al. Continuous flow-driven inversion for arterial spin labeling using pulsed radio frequency and gradient fields. Magn Reson Med 2008;60:1488-97

11. Alsop DC, Detre JA. Reduced transit-time sensitivity in noninvasive magnetic resonance imaging of human cerebral blood flow. J Cereb Blood Flow Metab 1996;16:1236-49

12. Wang J, Zhang Y, Wolf RL, et al. Amplitude-modulated continuous arterial spin-labeling 3.0-T perfusion MR imaging with a single coil: feasibility study. Radiology 2005;235:218-28

13. Jarnum H, Steffensen EG, Knutsson L, et al. Perfusion MRI of brain tumours: a comparative study of pseudo-continuous arterial spin labelling and dynamic susceptibility contrast imaging. Neuroradiology 2010;52:307-17

14. Treyer V, Jobin M, Burger C, et al. Quantitative cerebral H2(15)O perfusion PET without arterial blood sampling, a method based on washout rate. Eur J Nucl Med Mol Imaging 2003;30:572-80

15. Arigoni M, Kneifel S, Fandino J, et al. Simplified quantitative determination of cerebral perfusion reserve with $\mathrm{H} 2(15) \mathrm{O}$ PET and acetazolamide. Eur J Nucl Med 2000;27:1557-63

16. Hammers A, Allom R, Koepp MJ, et al. Three-dimensional maxi- mum probability atlas of the human brain, with particular reference to the temporal lobe. Hum Brain Mapp 2003;19:224-47

17. R Core Team. R: A language and environment for statistical computing. R Vienna, Austria: Foundation for Statistical Computing. http://www.R-project.org/.2013

18. Calamante F, Ganesan V, Kirkham FJ, et al. MR perfusion imaging in Moyamoya syndrome: potential implications for clinical evaluation of occlusive cerebrovascular disease. Stroke 2001;32:2810-16

19. Huisman TA, Sorensen AG. Perfusion-weighted magnetic resonance imaging of the brain: techniques and application in children. Eur Radiol 2004;14:59-72

20. Wang DJ, Alger JR, Qiao JX, et al. The value of arterial spin-labeled perfusion imaging in acute ischemic stroke: comparison with dynamic susceptibility contrast-enhanced MRI. Stroke 2012;43: $1018-24$

21. Ye FQ, Berman KF, Ellmore T, et al. H(2)(15)O PET validation of steady-state arterial spin tagging cerebral blood flow measurements in humans. Magn Reson Med 2000;44:450-56

22. Noguchi T, Kawashima M, Irie H, et al. Arterial spin-labeling MR imaging in Moyamoya disease compared with SPECT imaging. Eur J Radiol 2011;80:e557-562

23. Calamante F, Thomas DL, Pell GS, et al. Measuring cerebral blood flow using magnetic resonance imaging techniques. J Cereb Blood Flow Metab 1999;19:701-35

24. Wu WC, St Lawrence KS, Licht DJ, et al. Quantification issues in arterial spin labeling perfusion magnetic resonance imaging. Top Magn Reson Imaging 2010;21:65-73 